which survives to this day. At that time the articles were written in Latin, still the lingua franca of medical men, at least on the Continent. Today British readers can be grateful that English has attained an almost similar status in scientific communication, and nearly all the articles in the Acta are in our language. An effort was made from the start " to maintain the journal on a high scientific level and with diversified contents." The many British readers who consult it will join with us in congratulating its editors on continuing to achieve that aim today.

\section{Treatment of Hyperlipidaemia}

The term hyperlipidaemia implies an excess of lipid substances in the blood. Plasma lipids comprise free and esterified cholesterol, triglycerides, and phospholipids, together with a small but metabolically active free fatty acid fraction. Levels of plasma cholesterol and triglyceride can be readily estimated by conventional chemical techniques and are invariably raised, singly or in combination, in all hyperlipidaemic states. Lipids in the plasma are bound to protein, and these lipoproteins may be separated and characterized electrophoretically and by ultracentrifugation. Knowledge of the lipoprotein fraction present has proved invaluable in classifying and understanding the hyperlipidaemias.

Raised levels of plasma lipids are frequently found in a number of conditions-notably diabetes mellitus, the nephrotic syndrome, pancreatitis, and alcoholism. The treatment of these secondary hyperlipidaemias is that of the primary disease.

The primary hyperlipidaemias comprise a number of diseases with a genetic basis. The syndromes associated with them overlap to some extent, so that an exact classification of these disorders. has not yet been achieved. Xanthomata may make the hyperlipidaemic patient consult a dermatologist, or premature atherosclerosis may take him to a physician with cardiovascular complications. But the diagnosis may be made in an overtly healthy person whose serum cholesterol is estimated in the course of a routine medical examination.

Treatment of hyperlipidaemia is often unsatisfactory. This may be because of difficulty in reaching a precise diagnosis or because of the failure of some hyperlipidaemias to respond to the available treatment. Moreover, there may be some uncertainty about the usefulness of embarking on lifelong therapy the value of which in reducing cardiovascular complications is not clearly established.

The classification of both primary and secondary hyperlipidaemias has been placed on a more rational basis by $D$. S. Fredrickson and colleagues. ${ }^{1}$ They divided patients with hyperlipidaemia into five groups on the basis of lipoprotein analysis by electrophoresis and analytical ultracentrifugation. It seems probable that a classification on this basis will replace such terms as essential familial hypercholesterolaemia, ${ }^{2}$ essential hyperlipaemia, ${ }^{3}$ and fat-induced and carbohydrateinduced hyperlipaemia. ${ }^{4}$

Assessment of the effects of treatment should ideally include quantitative measurements of the main plasma lipoprotein fractions. E. H. Strisower and colleagues ${ }^{5}$ have recently reported such a study. They also divided their patients into five groups on the basis of lipoprotein analysis, but their groups differed in some respects from those of Fredrickson and his colleagues. ${ }^{1}$ Group 1 showed raised Sf $0-12$ lipoprotein levels ( $\beta$ lipoprotein; low-density lipoproteins) with normal or low Sf $20-400$ levels (pre- $\beta$ lipoprotein; very low density lipoproteins). All patients in this group had tendon xanthomata and many showed arcus senilis. Group 2 patients had a similar lipoprotein pattern but did not have tendon xanthomata and differed in their response to clofibrate. Groups 1 and 2 correspond to Fredrickson's type II hyperlipoproteinaemia. Patients in group 3 showed an increase of Sf 12-20 and Sf 20-400 levels, together with tuberous xanthomata. Group 4 comprised patients with raised Sf $20-400$ levels, moderately low Sf 0-12 and normal Sf 12-20 levels. Localized tuberous xanthomatous lesions were not seen in this group, but transient eruptive xanthomata occasionally developed in association with severe diabetes. Group 5 included patients with raised Sf $0-20$ and Sf $20-400$ levels. Strisower and his colleagues did not have any patients with the rare condition of fatinduced hyperlipoproteinaemia in their study.

The five groups differed in their response to treatment. The different types of treatment given were supplementary corn oil, carbohydrate restriction, thyroid preparations, and clofibrate. Patients in Group 1 did not respond to diet or to clofibrate, but showed moderate falls in Sf 0-20 levels with large doses of D- and L-thyroxine. Group 2 patients differed from Group 1 in responding moderately to clofibrate and to thyroid. Group 3 patients showed an excellent response to clofibrate, and this was considered to be the best treatment for this group. Group 4 patients showed large falls in Sf 20 400 levels both with carbohydrate restriction and with clofibrate, but the latter drug produced concomitant rises in Sf 0-20 levels. Because of this finding, carbohydrate restriction was considered preferable in this group. Group 5 patients responded best to combined therapy with thyroid and clofibrate.

The study thus confirmed earlier observations that raised $\beta$ or low-density lipoprotein levels are relatively refractory to treatment with clofibrate, while raised pre- $\beta$ or very lowdensity lipoproteins respond well to both carbohydrate restriction and clofibrate. ${ }^{4} 7$ This must be qualified by the interesting observation that some patients showed concomitant rises in Sf 0-20 levels an clofibrate. Some evidence suggests that this lipoprotein fraction may be more atherogenic than the very low-density lipoproteins. ${ }^{8}$

Studies of this type depend on ultracentrifugation, a technique not available to most physicians concerned with the treatment of hyperlipidaemia. However, a working diagnosis may usually be reached by a clinical assessment of the type and distribution of xanthomata, if present ; by examination of plasma for lactescence after a meal; by estimation of plasma cholesterol and triglyceride, with calculation of the

1 Fredrickson, D. S., Levy, R. I., and Lees, R. S., New Engl. F. Med., $1967,276,34,94,148,215,273$

Wilkinson, C. F., Hand, E. A., and Fliegelman, M. T., Ann. intern. Med., 1948, 29, 671.

Harrison M. T. and Goldberg, D. M., F. Atheroscler. Res., 1963, 3,

561 .
Ahrens, E. H., Hirsch, J., Oette, K., Farquhar, J. W., and Stein, Y., Trans. Ass. Amer. Phycns, 1961, 74, 134.

Trans. Ass. Amer. Phycns, 1961, 74, 134 .
Strisower, E. H., Adamson, G., and Strisower, B., Amer. F. Med., 1968, 45, 488.

- Howard, R. P., Alaupovic, P., Brusco, O. J., and Furman, R. H., f. Atheroscler. Res., 1963, 3, 482.

Strisower, E. H., F. Atheroscler. Res., 1963, 3, 445.

Gofman, J. W., Young, W., and Tandy, R., Circulation, 1966, 34, 679. - Lees, R. S., and Hatch, F. T., F. Lab. clin. Med., 1963, 61, 518

Brit. med. f., 1968, 3, 689.

M.R.C. Research Committee, Lancet, 1968, 2, 693

2 Leren, P., Acta med. scand., 1966, suppl. 466. 
cholesterol/triglyceride ratio $^{5}$; and by paper electrophoresis of plasma lipoproteins. ${ }^{9}$ Treatment by diet or by drugs can best be assessed by the individual patient's response, the physician using guide lines such as those laid down by Strisower and his colleagues. ${ }^{5}$ It is clear from their study that the use of more than one form of treatment may be necessary to obtain optimum results. The management of the hyperlipidaemic patient should always include a search for affected relatives.

What are the indications for treatment? In patients with xanthomatosis a reduction in the hyperlipidaemia is usually followed by a disappearance of the lesions, and the cosmetic result alone makes treatment worth while. But the main reason for treating patients with hyperlipidaemia is to prevent the development of atherosclerosis and so reduce the incidence of cardiovascular complications. So far treatment has not been conclusively proved to achieve this. Though the preliminary results of clinical trials of prophylactic treatment are encouraging, more information is required before any of the forms of therapy mentioned above can be recommended on clinical grounds. ${ }^{10}$ Clinical trials of treatment in patients with hyperlipidaemia who have already sustained a cardiovascular lesion have given conflicting results. ${ }^{11} 12$

\section{Strokes and the Pill}

Neurological disorders which may possibly be caused by the oral contraceptives continue to stimulate interest and engender controversy. Attacks of cerebrovascular occlusion or deficiency, being the most dramatic and potentially dangerous, have received most attention. The evidence for a causal connexion has ranged from the strong, if not very adequately controlled, association reported by E. R. Bickerstaff and J. McD. Holmes, ${ }^{1}$ whose study appeared clearly to link the pill with episodes of cerebrovascular insufficiency in otherwise healthy young women, through the similar, but somewhat less dogmatic, studies of L. Illis and his colleagues, ${ }^{2}$ to the failure of W. B. Jennett and J. N. Cross ${ }^{3}$ to find any such association in their study of ischaemic attacks in the territory of the carotid artery. The Committee on the Safety of Drugs has moved from its early cautious view of $1965^{4}$ that as yet evidence was insufficient that such episodes did more than represent their natural occurrence in women of childbearing age, through the preliminary report of a further study in $1967^{\circ}$ which referred to " a relation" between deaths from pulmonary embolism or infarction and the use of oral contraceptives, and possibly also with cerebral thrombosis, to their findings in $1968^{6}$ of a "strong relation" with deaths from these conditions. At the same time from the M.R.C.'s statistical research unit" came the conclusion that "oral contraceptives can also be a cause of cerebrovascular insufficiency."

Arguments following such publications have tended to centre on the advisability of allowing the " pill " to be used at all, and insufficient attention has been given to the definition of those groups of patients who, among the many thousands safely using oral contraception, are at risk of developing neurological complications. From whom should they be withheld, and from whom should they be withdrawn? It is generally agreed that previous thromboembolism, liver or renal disease, and blood diseases are contraindications, and Bickerstaff and Holmes ${ }^{1}$ considered that anything suggestive of a transient ischaemic attack should be an indication for immediate withdrawal of the drug. They also advised that a change in the character of pre-existing migrainous attacks should probably be a further warning sign. M. L. Salmon, J. Z. Winkelman, and A. J. Gay ${ }^{8}$ have now made another attempt to clarify the problem by analysing, from the literature, 100 patients on oral contraceptives who had developed neurological or ophthalmic disorders, adding four of their own cases, and 129 with migraine. Special attention was paid to any premonitory symptoms. They found that out of 70 reported "strokes," though 52 were clinically in the territory of the middle cerebral artery, 18 (25\%) were in the vertebrobasilar territory, and they state that they could find no record of vertebrobasilar occlusion in otherwise healthy young women except in those who had been on the pill. They were convinced also that migraine can be caused or worsened, and mention reports of a number of miscellaneous conditions of which " optic neuritis," retinal vascular lesions, and pseudotumor cerebri (benign intracranial hypertension) are the most convincing. They considered that, from studying the previous history of women on oral contraceptives who developed major neurological disorders, the majority (52 out of 87) had shown evidence in the past of hypertension, some other form of vascular disease, migraine, or endometriosis.

As the use of the pill becomes more widespread throughout the world it is clear that this aspect of the problem must be investigated further, not only to ensure that the woman at risk is protected so far as is possible, but also that indiscriminate stopping of what is a highly effective contraceptive method does not follow every vague disturbance which might possibly be referable to the nervous system. No dogmatic guidance is possible, but experience suggests that previous thromboembolism in any part of the body, hypertension, and severe migraine should probably contraindicate the use of oral contraceptives. Any hint, during their administration, of transient cerebral or retinal ischaemia should demand their immediate withdrawal. A change in a migrainous patient from a diffuse type of attack to a strongly focal one, suggesting a disturbance of the behaviour of a particular arterial territory, should be taken as a timely warning that some effect -what, is not yet known-is probably occurring in the cerebral vasculature, and under these circumstances discretion is surely the better part of valour. Nor is it clear which component of the large number of oral contraceptives now available is responsible for neurological side-effects, and so great is the variety of these preparations that it has been difficult to observe sufficient recorded attacks on each from which to draw conclusions. It is therefore most important that the reporting of these side-effects should continue. It is only on a nationwide basis that significant figures can be obtained, and if, for instance, the small oestrogen or continuous progestogen dosage schedules could be shown truly to reduce the number of neuro-ophthalmic side-effects, then a great step forward towards safety and confidence would have been taken. So far such information is not available.

1 Bickerstaff, E. R., and Holmes, J. McD., Brit. med. F., 1967, 1, 726

2 Illis, L., Kocen, R. S., McDonald, W. I., and Mondkar, V. P., Brit. med. F., 1965, 2, 1164 .

3 Jennett, W. B., and Cross, J. N., Lancet, 1967, 1, 1019.

4 Cahal, D. A., Brit. med. F., 1965, 2, 1180.

Brit. med. F., 1967, 2, 355.

I Inman, W. H. W., and Vessey, M. P., Brit. med. f., 1968, 2, 193.

- Vessey, M. P., and Doll, R., Brit. med. f., 1968, 2, 199.

${ }^{8}$ Salmon, M. L., Winkelman, J. Z., and Gay, A. J., f. Amer. med. Ass., 1968, 206, 85 . 